\title{
Appendix 2: OECD Global Science Forum, Task Force on Radio Astronomy and the Radio Spectrum
}

\author{
Terms of Reference, March 2001
}

\section{Background}

The deployment of new low-orbiting telecommunications satellites will have a major impact on the future of radio astronomy. Radio telescopes are extremely sensitive, and signals from telecommunications satellites can overwhelm the signals from astronomical sources, thus preventing scientists from collecting data. For example, a simple portable telephone, if placed on the Moon, would be among the brightest astronomical objects, as seen from Earth. In the past, radio astronomers have sought protection from man-made signals by placing their telescopes in remote locations, These measures may no longer permit scientific observations, since the new satellites are designed to provide $100 \%$ global coverage. Also, radio astronomers made their observations in the frequency windows reserved for their exclusive use by the International Telecommunications Union. However, astronomers have now discovered that signals from the most distant and exotic objects in the sky are shifted in frequency due to the expansion of the Universe - and hence must be observed outside the bandwidths reserved for science.

To ensure that the science of radio astronomy can flourish along with a thriving telecommunications industry, new innovative and effective measures to permit astronomical signal detection will need to be developed before the next generation of radiotelescopes will be built. These instruments, which will be very few in number, will be a hundred times more sensitive than current telescopes - sensitive enough to permit observation of nearly the entire extent and history of the observable Universe.

The need to reconcile and support the interests of both the telecommunications industry and the radio astronomy community was highlighted in the report of a working group of the OECD Megascience Forum, now referred to as the OECD Global Science Forum, and was discussed by the Science Ministers of the OECD countries at their meeting in June 1999. In the communique of the meeting, the Ministers endorsed the establishment of an informal high-level Task Force to map out a strategy for ensuring the future of radio astronomy, while allowing for the continued vigorous growth of commercial space-based telecommunications.

The Task Force should have approximately ten members, among them senior executives of telecommunications companies, members of the scientific community, and persons who are active in national and international regulatory bodies. Task Force members should be leaders in their fields, with a strategic view of future developments, an appreciation of the value of astronomy and of telecommunications, and a desire to find win-win solutions for the scientific and business communities. They will participate in a personal capacity; however, they will be expected to maintain contacts with their organisations and their professional colleagues, to ensure openness and balance in the deliberations of the group. The Task Force is expected to complete its work in approximately 
one year, with two meetings convened during that time. Detailed studies by experts could be commissioned as needed.

\section{Objectives}

The Task Force should produce a brief, policy-level report that contains findings and action-oriented recommendations. While the Task Force is expected to formulate a long-term strategic plan, it should, whenever possible, propose specific activities that can be undertaken by existing national and international organisations and institutions that are already active in this area, or have the authority to act and generate concrete results. It is recognised that the ultimate resolution of this difficult problem could take many years, but the deliberations of the Task Force should lead to the initiation of appropriate activities in the near future. Examples of such activities could include: co-operative R\&D projects involving satellite manufacturers and radio astronomy laboratories, analysis and discussion of regulatory and technical issues by ITU working parties, and feasibility studies by international bodies for new agreements or treaties. As it proceeds, the Task Force may wish to provide progress reports to the OECD Global Science Forum, the successor to the Megascience Forum. This committee consists of senior government science policy officials who will be available to provide a governmental perspective on the ongoing work of the Task Force.

\section{Scope and Work Program}

While the Task Force will be free to define its own work programme, it might choose to examine three general areas that were previously identified by the working group of the Megascience Forum:

A. Technological solutions: Radio astronomers and industry representatives could identify and jointly implement interference mitigation schemes.

B. Regulation: If new, innovative ways of sharing the radio spectrum are to be found, the appropriate discussions must begin soon within national agencies and the ITU, as the implementation of new regulations usually requires a considerable amount of effort over several years.

C. Radio-quiet zones: Remote areas on the Earth's surface could be designated where future radio observatories could be located, and where radio emissions, especially from spaceborne and airborne sources, would be restricted in frequency and time. The technical, regulatory, and legal dimensions of this concept would have to be carefully examined.

Accordingly, the Task Force could choose:

A. To investigate in detail the three-pronged approach indicated above, and to propose specific programs that will constitute a balanced, co-ordinated strategy.

B. To sound out members of their respective spheres of interest about the elements of acceptable regulatory and other solutions, and about their willingness to participate in achieving those solutions.

C. To develop a road map of long-term solutions, with a time schedule and milestones, for consideration by the relevant national and international bodies. Special attention should be given to taking advantage of (and strengthening) the procedures of the ITU. 